\title{
Outcomes of neonates requiring prolonged stay in the intensive care unit after surgical repair of congenital heart disease
}

\author{
Makoto Mori, MD, ${ }^{\mathrm{a}}$ Courtney McCracken, $\mathrm{PhD},{ }^{\mathrm{b}}$ Kevin Maher, MD, ${ }^{\mathrm{b}}$ Brian Kogon, MD, ${ }^{\mathrm{a}}$ \\ William Mahle, MD,${ }^{b}$ Kirk Kanter, MD, ${ }^{a}$ and Bahaaldin Alsoufi, MD ${ }^{a}$
}

\begin{abstract}
Objective: After neonatal cardiac surgery, a number of patients need a prolonged stay in the intensive care unit (ICU). Those patients require tremendous resources and strain the capacity of cardiac units. To date, little knowledge of early and late survival for this challenging population exists.

Methods: From 2002 to 2012, 108 neonates required a postoperative ICU stay $>30$ days. Multivariable regression analyses examined factors associated with hospital death and late survival. Comparison of late outcomes in hospital survivors was made between those who had prolonged ICU stay $(\mathrm{n}=82)$ and contemporaneous neonates who did not $(\mathrm{n}=1329)$.
\end{abstract}

Results: Hospital mortality occurred in 26 of 108 patients (24\%). On multivariable analysis, factors associated with mortality were use of extracorporeal membrane oxygenation (odds ratio, 3.4 [95\% confidence interval, 1.3-9.1], $P=.014$ ) and renal failure that required dialysis (odds ratio, $3.1[95 \%$ confidence interval, 1.0-10.0], $P=.056$ ). Overall survival at 1 and 8 years was $57 \%$ and $51 \%$. Comparison of late outcomes for hospital survivors showed that neonates who required prolonged postoperative stay in the ICU had significantly worse 8 -year survival $(69 \%$ vs $92 \% ; P<.001)$ and that the effect of prolonged stay in the ICU on diminished survival was more pronounced in neonates with 2 ventricles ( $68 \%$ vs $95 \%$, hazard ratio, 8.0 [95\% confidence interval, 4.2-15.1], $P<.001)$ than in those with single ventricle $(66 \%$ vs $81 \%$; hazard ratio, 2.0 [95\% confidence interval, 1.1-3.5], $P=.021$ ). Overall, 77\% of single-ventricle hospital survivors who required prolonged stay in the ICU progressed to Glenn, with $82 \%$ of them reaching or qualifying for subsequent Fontan.

Conclusions: Prolonged postoperative stay in the ICU is associated with high hospital and significant postdischarge mortality, mainly during the first year. In neonates with single ventricle, prolonged stay in the ICU was associated with high hospital and interstage mortality and usual progression subsequent to Glenn shunt. In contrast, prolonged stay in the ICU in neonates with 2 ventricles was associated with high hospital mortality and considerable decrease in late survival, suggesting a more pronounced deviation from expected survival in those patients. (J Thorac Cardiovasc Surg 2016;152:720-7)

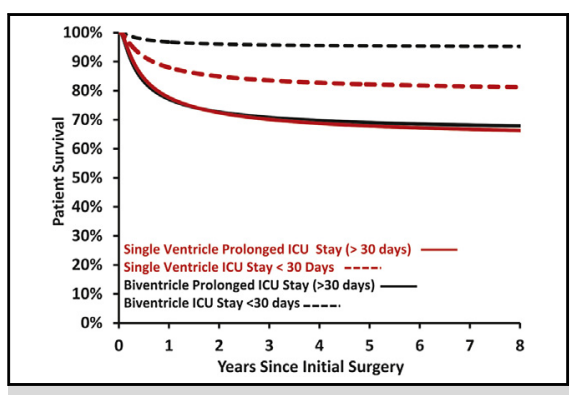

Effect of prolonged stay in the intensive care unit on postdischarge survival in single and 2 ventricle neonates.

\section{Central Message}

Prolonged postoperative stay in the intensive care unit increases postdischarge mortality, especially in neonates with 2 ventricle anomalies.

\section{Perspective}

Prolonged postoperative stay in the intensive care unit (ICU) is associated with high hospital and postdischarge mortality. In single-ventricle patients, prolonged stay in the ICU is associated with high hospital mortality and usual progression subsequent to Glenn shunt. Conversely, prolonged stay in the ICU in 2-ventricle patients is associated with high hospital mortality and considerable decrease in late survival.

See Editorial Commentary page 727.
From the Divisions of a Cardiothoracic Surgery and ${ }^{\mathrm{b}}$ Cardiology, Children's Healthcare of Atlanta, Emory University School of Medicine, Atlanta, Ga.

Read at the 41st Annual Meeting of The Western Thoracic Surgical Association, Whistler, British Columbia, Canada, June 24-27, 2015.

Received for publication Sept 14, 2015; revisions received April 8, 2016; accepted for publication April 13, 2016; available ahead of print May 10, 2016.

Address for reprints: Bahaaldin Alsoufi, MD, Division of Cardiothoracic Surgery, Emory University School of Medicine, Children's Healthcare of Atlanta, 1405 Clifton Rd, NE, Atlanta, GA 30322 (E-mail: balsoufi@hotmail.com).

0022-5223/\$36.00

Copyright (c) 2016 by The American Association for Thoracic Surgery

http://dx.doi.org/10.1016/j.jtcvs.2016.04.040
The advances in operative management and perioperative care have allowed the successful treatment of neonates born with complex congenital heart disease. Subsequently,

Scanning this QR code will take you to the supplemental table for this article. 


$$
\begin{aligned}
& \text { Abbreviations and Acronyms } \\
& \begin{aligned}
\mathrm{CI} & =\text { confidence interval } \\
\mathrm{ECMO} & =\text { extracorporeal membrane oxygenation } \\
\mathrm{HR} & =\text { hazard ratio } \\
\mathrm{ICU} & =\text { intensive care unit } \\
\mathrm{IQR} & =\text { interquartile range }
\end{aligned}
\end{aligned}
$$

an increasing number of neonates who undergo cardiac surgery require prolonged stay in the intensive care unit (ICU). Chronic critical illness is defined in the pediatric and adult literature as duration of stay in the ICU longer than 28-30 days. ${ }^{1-5}$ Although it's natural that hospital mortality is increased in patients with chronic critical illness, little is known about the late outcomes of those patients subsequent to hospital discharge, especially in the pediatric cardiac population. In adult cardiac patients, the effect of prolonged postoperative stay in the ICU has been found to be associated with reduced late survival, low quality of life, and high rates of readmission. ${ }^{1-5}$

Neonates who need prolonged ICU care require tremendous resources and strain the capacity of cardiac units. Although previous studies have identified risk factors associated with prolonged stay in the ICU after cardiac surgery, ${ }^{6-9}$ the impact of prolonged stay in the ICU on the long-term prognosis of pediatric cardiac population remains unclear.

We aim in the current series to describe the characteristics of neonates who required prolonged ICU care after cardiac surgery at our institution, to examine early and late results and risk factors associated with survival, and finally to compare late outcomes with a control of contemporaneous neonates who survived hospitalization without requiring prolonged ICU stay.

\section{PATIENTS AND METHODS \\ Inclusion Criteria}

We examined outcomes of neonates who underwent cardiac surgery at Children's Healthcare of Atlanta, Emory University from 2002 to 2012. Premature neonates who received patent ductus arteriosus closure and children who were in heart failure supported by ventricular assist device were excluded. Patients were identified with the use of our institutional surgical database. Demographic, morphologic, clinical, operative, and hospital details were abstracted from the medical records for analysis. Approval of this study was obtained from our hospital's Institutional Review Board, and requirement for individual consent was waived for this observational study.

\section{Follow-up}

Time-related outcomes were determined from recent office visits documented in the electronic chart of Children's Healthcare of Atlanta system or from direct correspondence with pediatric cardiologists outside the system. Mean follow-up duration was $3.0 \pm 3.45$ years and was $95 \%$ complete.

\section{Statistical Analysis}

Statistical analyses were performed with SAS 9.3 (SAS Institute, Inc, Cary, NC), and statistical significance was assessed at the .05 level of significance. Data are presented as medians with interquartile ranges (IQRs) or frequencies and percentages as appropriate. Comparisons among neonates who required a prolonged stat in the ICU and who died before hospital discharge with those who survived hospital discharge were made by the use of $\chi^{2}$ tests, Fisher exact tests for categorical data, and Mann-Whitney $U$ tests or Kolmogorov-Smirnov tests for continuous data. Similar analyses also were performed to compare characteristics of neonates who required a prolonged postoperative stay in the ICU with those who did not. On the basis of univariate analyses, variables significant at the 0.2 level were entered into a multivariable logistic regression model to identify predictors of mortality after surgery. Variable were retained in the multivariable logistic model if they were significant at the 0.1 level. The time-dependent outcomes (overall survival since surgery and since hospital discharge) were parametrically modelled with the HAZARD procedure in SAS. Parametric probability estimates for time-dependent outcomes uses models based on multiple, overlapping phases of risk (available for use with the SAS system at https://www.lerner.ccf.org/qhs/ software/hazard). The HAZARD procedure uses maximum likelihood estimates to resolve risk distribution of time to event in up to 3 phases of risk (early, constant, and late). These parametric hazard models were created for overall mortality and mortality conditional on surviving hospital discharge after surgery. To examine the effect of prolonged ICU stay and single-ventricle anomalies on survival, models were stratified by prolonged ICU status and ventricle anomaly, and survival probabilities are reported for each group. The effect of prolonged stay in the ICU on subsequent progression to Glenn and Fontan after the initial surgery was examined with the use of $\chi^{2}$ tests.

\section{RESULTS}

Patients' Characteristics, Morphologic, and Operative Details of the Prolonged ICU Stay Cohort

On the basis of the definition of chronic critical illness, we used ICU stay $>30$ days as our cutoff to describe patients who required prolonged postoperative ICU stay. Between 2002 and 2012, 171 children required prolonged postoperative ICU stay $>30$ days at our institution. This number represents $\sim 3 \%$ of all children who were admitted to our ICU after surgical repair of their congenital heart disease. We focused our current study on neonates. During this study interval, 1538 neonates underwent cardiac surgery at our institution; our prolonged ICU cohort comprised 108 neonates $(\sim 7 \%)$ who required postoperative ICU stay $>30$ days.

There were 58 male patients (54\%), and median age at surgery was 5 days (IQR, 3-9). Mean weight was $3.0 \pm 0.6 \mathrm{~kg}$ with 20 patients $(19 \%) \leq 2.5 \mathrm{~kg}$ at time of surgery. There were 22 patients $(21 \%)$ who were born prematurely $\leq 36$ weeks' gestation. Fifty patients $(46 \%)$ had associated genetic syndromes and/or major extracardiac malformations, including heterotaxy syndrome $(\mathrm{n}=16)$, DiGeorge syndrome $(\mathrm{n}=12)$, CHARGE (ie, Coloboma of the eye, Heart defects, Atresia of the choanae, Retardation of growth and/ or development, Genital and/or urinary abnormalities, and Ear abnormalities and deafness) syndrome $(\mathrm{n}=4)$, VACTERL (ie, costo-Vertebral abnormalities, Anal atresia, Cardiac defects, Tracheal-esophageal abnormalities, Renal and radial abnormalities, nonradial Limb abnormalities) association $(\mathrm{n}=3)$, Down syndrome $(\mathrm{n}=2)$, Dandy Walker syndrome $(\mathrm{n}=2)$, and other $(\mathrm{n}=11)$, Table 1 . 
TABLE 1. Patient characteristics, anatomic, and operative details of neonates who required prolonged stay in the ICU after cardiac surgery with comparison between hospital survivors and nonsurvivors

\begin{tabular}{|c|c|c|c|c|}
\hline \multirow[b]{2}{*}{ Characteristic } & \multirow[b]{2}{*}{ Overall $(\mathbf{N}=108)$} & \multicolumn{2}{|c|}{ Hospital survival } & \multirow[b]{2}{*}{$P$ value } \\
\hline & & Yes (alive) $(\mathbf{n}=\mathbf{8 2})$ & No $($ dead $)(n=26)$ & \\
\hline Male sex & $58(54 \%)$ & $43(52 \%)$ & $15(58 \%)$ & 64 \\
\hline Age at surgery $(\mathrm{d})$, median (IQR) & $5(3-9)$ & $5(3-9)$ & $5(2-10)$ & .49 \\
\hline Premature $\leq 36 \mathrm{wk}$ & $22(21 \%)$ & $18(23 \%)$ & $4(15 \%)$ & .44 \\
\hline Genetic syndromes and extracardiac malformations & $50(46 \%)$ & $39(48 \%)$ & $11(42 \%)$ & .64 \\
\hline Weight $(\mathrm{kg})$, mean $\pm \mathrm{SD}$ & $3.0 \pm 0.6$ & $3.0 \pm 0.6$ & $3.1 \pm 0.5$ & .51 \\
\hline Weight $\leq 2.5 \mathrm{~kg}$ & $20(19 \%)$ & $16(20 \%)$ & $4(15 \%)$ & .64 \\
\hline Underlying cardiac anomaly & & & & .08 \\
\hline Single ventricle & $63(58 \%)$ & $44(54 \%)$ & $19(73 \%)$ & \\
\hline Two ventricles & $45(42 \%)$ & $38(46 \%)$ & $7(27 \%)$ & \\
\hline STAT category 4 and 5 & $92(85 \%)$ & $70(85 \%)$ & $22(85 \%)$ & .93 \\
\hline Use of cardiopulmonary bypass & $90(83 \%)$ & $67(82 \%)$ & $23(89 \%)$ & .42 \\
\hline Cardiopulmonary bypass duration ( $\mathrm{min}$ ), median (IQR) & $139(71-166)$ & $140(81-169)$ & $127(60-160)$ & .406 \\
\hline Postoperative use of ECMO & $30(28 \%)$ & $17(21 \%)$ & $13(50 \%)$ & .004 \\
\hline Unplanned reoperation & $24(22 \%)$ & $17(21 \%)$ & $7(27 \%)$ & .51 \\
\hline Sepsis* & $38(36 \%)$ & $27(34 \%)$ & $11(42 \%)$ & .43 \\
\hline Renal failure* & $16(15 \%)$ & $8(10 \%)$ & $8(31 \%)$ & .01 \\
\hline Cerebrovascular accident* & $7(7 \%)$ & $5(6 \%)$ & $2(8 \%)$ & 1.00 \\
\hline Necrotizing enterocolitis* & $18(17 \%)$ & $15(19 \%)$ & $3(12 \%)$ & .39 \\
\hline Gastrointestinal bleeding* & $4(4 \%)$ & $2(3 \%)$ & $2(8 \%)$ & .25 \\
\hline Chylothorax* & $22(21 \%)$ & $17(21 \%)$ & $5(19 \%)$ & .83 \\
\hline Vocal cord paralysis* & $4(4 \%)$ & $4(5 \%)$ & $0(0 \%)$ & .25 \\
\hline
\end{tabular}

$I Q R$, Interquartile range; $S D$, standard deviation; STAT, Society of Thoracic Surgeons-European Association for Cardio-Thoracic Surgery Congenital Heart Surgery mortality categories; $E C M O$, extracorporeal membrane oxygenation. *Data present for 106 of 108 patients.

Sixty-three patients (58\%) had underlying singleventricle anomalies. At time of the index admission, 90 patients $(83 \%)$ underwent open cardiac procedures that required the use of cardiopulmonary bypass, whereas the remaining $18(17 \%)$ underwent closed cardiac procedures. After their cardiac procedure, 30 patients $(28 \%)$ required extracorporeal membrane oxygenation (ECMO) support and $24(22 \%)$ required unplanned cardiac reoperation (surgical revisions not including delayed sternal closure, mediastinal explorations, ECMO procedures, or procedure to treat noncardiac complications such as diaphragm plication or thoracic duct ligation), Table 1. Median postoperative ventilation, ICU, and hospital stay were 27.6 (IQR, 15.3-42.0), 44.7 (IQR, 36.0-60.0), and 54.0 (IQR, 44.0-76.0) days, respectively.

\section{Survival of the Prolonged ICU Stay Cohort}

Overall, hospital mortality occurred in 26 patients $(24 \%)$. The differences in demographic, morphologic, and operative characteristics between hospital survivors and nonsurvivors are detailed in Table 1. On multivariable analysis, factors associated with hospital mortality were use of ECMO (odds ratio, 3.4 [95\% confidence interval (CI), 1.3-9.1], $P=.014$ ) and renal failure requiring dialysis (odds ratio, 3.1 [95\% CI, 1.0-10.0], $P=.056$ ).

Subsequent to hospital discharge, there were 30 additional deaths $(28 \%)$, with the majority of those additional deaths $(n=22,73 \%)$ occurring within the first year after surgery and all occurring within 3 years from surgery. On importance, 28 of 30 late deaths were labeled cardiac, with 17 patients presenting with cardiac arrest at time of their death. Overall survival for the entire cohort was $57 \%$ at 1 year and $52 \%$ at 5 years after surgery, Figure 1. No factors were identified to be associated with the risk of death after hospital discharge, Table E1.

\section{Comparison of Postdischarge Survival Between Neonates Who Required Prolonged Stay in the ICU and Those Who Did Not}

We studied the effect of ICU stay as a continuous variable on postdischarge survival in all neonates who survived initial hospitalization after cardiac surgery $(\mathrm{n}=1411)$. Longer stay in the ICU was associated with decreased survival after hospital discharge (log 2 ICU stay: hazard ratio [HR], 1.7 [95\% CI, 1.5-1.9], $P<.001)$. After adjusting for other established risk factors for mortality after neonatal cardiac surgery (ie, single-ventricle anomaly, low weight $[\leq 2.5 \mathrm{~kg}]$, prematurity $\leq 36$ weeks, genetic syndromes and extracardiac anomalies, use of cardiopulmonary bypass, and the Society of Thoracic SurgeonsEuropean Association for Cardio-Thoracic Surgery Congenital Heart Surgery mortality categories), we found that longer stay in the ICU continued to be associated with decreased survival after hospital discharge in the adjusted analysis ( $\log 2$ ICU stay: HR, 1.5 [95\% CI, 1.3-1.7], $P<.001)$. 

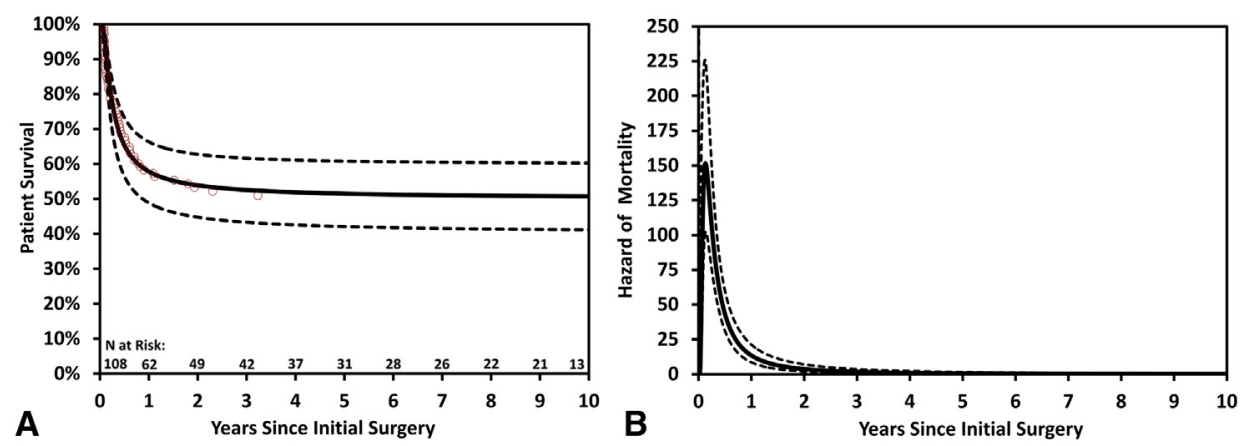

FIGURE 1. A, Time-dependent survival and B, risk hazard of death over time after index surgical procedure in 108 neonates who required prolonged postoperative ICU stay. The solid lines in the parametric model represent parametric point estimates, and the dashed lines enclose the $95 \%$ confidence interval. Circles represent nonparametric estimates.

We compared postdischarge survival of neonates who required prolonged ICU stay $>30$ days and those who did not to better assess the effect of prolonged ICU stay on late events. After exclusion of those who did not survive initial hospital admission, our comparison cohort was composed of 82 neonates who required prolonged ICU stay $>30$ days and 1329 neonates who did not require ICU stay $>30$ days. The differences in the demographic, morphologic, and operative variables between the 2 comparison groups are detailed in Table 2. Neonates who required prolonged stay in the ICU were more likely to be premature, have genetic syndromes, and/or extracardiac malformations and to have single-ventricle anomalies. In

TABLE 2. The differences in the demographic, morphologic, and operative variables between neonates who required prolonged stay in the ICU and those who did not after exclusion of hospital nonsurvivors

\begin{tabular}{|c|c|c|c|}
\hline Characteristic & $\begin{array}{c}\text { ICU stay } \\
\leq \mathbf{3 0} \mathbf{d} \\
(\mathbf{n}=\mathbf{1 3 2 9})\end{array}$ & $\begin{array}{c}\text { ICU stay } \\
>30 \text { d } \\
(n=82)\end{array}$ & $P$ value \\
\hline Age (d), median (IQR) & $6(3-10)$ & $5(3-9)$ & .537 \\
\hline Weight $(\mathrm{kg})$, mean $\pm \mathrm{SD}$ & $3.2 \pm 0.6$ & $3.0 \pm 0.6$ & .023 \\
\hline Weight $\leq 2.5 \mathrm{~kg}$ & $178(13 \%)$ & $16(20 \%)$ & .118 \\
\hline Male sex & $802(60 \%)$ & $43(52 \%)$ & .156 \\
\hline Premature $\leq 36 \mathrm{wk}$ & $174(13 \%)$ & $18(23 \%)$ & .020 \\
\hline $\begin{array}{l}\text { Genetic syndromes and } \\
\text { extracardiac malformations }\end{array}$ & $228(17 \%)$ & $39(48 \%)$ & $<.001$ \\
\hline \multicolumn{4}{|l|}{ Underlying cardiac anomaly } \\
\hline Single ventricle & $359(27 \%)$ & $44(54 \%)$ & $<.001$ \\
\hline Two ventricles & $970(73 \%)$ & $38(46 \%)$ & \\
\hline \multicolumn{4}{|l|}{ Initial procedure } \\
\hline Palliation & $534(40 \%)$ & $55(68 \%)$ & $<.001$ \\
\hline Repair & $794(60 \%)$ & $26(32 \%)$ & \\
\hline $\begin{array}{l}\text { Use of cardiopulmonary } \\
\text { bypass }\end{array}$ & $834(63 \%)$ & $67(82 \%)$ & $<.001$ \\
\hline Unplanned reoperation & $55(4 \%)$ & $17(21 \%)$ & $<.001$ \\
\hline ECMO requirement & $22(2 \%)$ & $17(21 \%)$ & $<.001$ \\
\hline
\end{tabular}

addition, they were more likely to have undergone unplanned cardiac reoperation or have required postoperative ECMO support. Those who required prolonged postoperative ICU stay $>30$ days had significantly worse later survival compared with those who did not: 1-year survival, $77 \%$ versus $94 \%$, and 8 -year survival, $69 \%$ versus $92 \%$, respectively $(P<.001)$, Figure 2 . Prolonged stay $>30$ days in the ICU was associated with decreased survival after hospital discharge (HR, 4.5 [95\% CI, 2.9-6.9], $P<.001$ ) on the unadjusted analysis and continued to be associated with decreased survival on the adjusted analysis (HR, 2.5 [95\% CI, 1.6-4.0], $P<.001$ ).

There was an interesting interaction between underlying cardiac anomaly (single-ventricle vs 2 ventricles) and prolonged ICU stay that is best depicted in Figure 3. Although prolonged stay in the ICU was associated with worse survival in both groups, the effect of prolonged stay in the ICU on diminished late survival was more pronounced in neonates with 2 ventricles (8-year survival $68 \%$ vs $95 \%$, HR, 8.0 [95\% CI, 4.2-15.1], $P<.001)$ than in those with single ventricle (8-year survival $66 \%$ vs $81 \%$, HR, 2.0 [95\% CI, 1.1-3.5], $P=.021$ ).

\section{Progress of Neonates With Single Ventricle Who Required Prolonged Stay in the ICU After First- Stage Palliation}

Comparison between single-ventricle hospital survivors who required a prolonged stay in the ICU after first-stage palliation versus those who did not was performed and is shown in Figure 4. The prolonged ICU stay cohort included 43 neonates $(11 \%)$, whereas the control group of singleventricle neonates who did not require prolonged ICU stay included 358 neonates $(89 \%)$. Neonates who required prolonged ICU stay were less likely to progress to subsequent Glenn shunt $(77 \%$ vs $88 \%, P=.037)$ and more likely to die or require heart transplantation before Glenn shunt ( $23 \%$ vs $11 \%, P=.013)$. Subsequent to Glenn shunt, there was a trend in those who required prolonged 

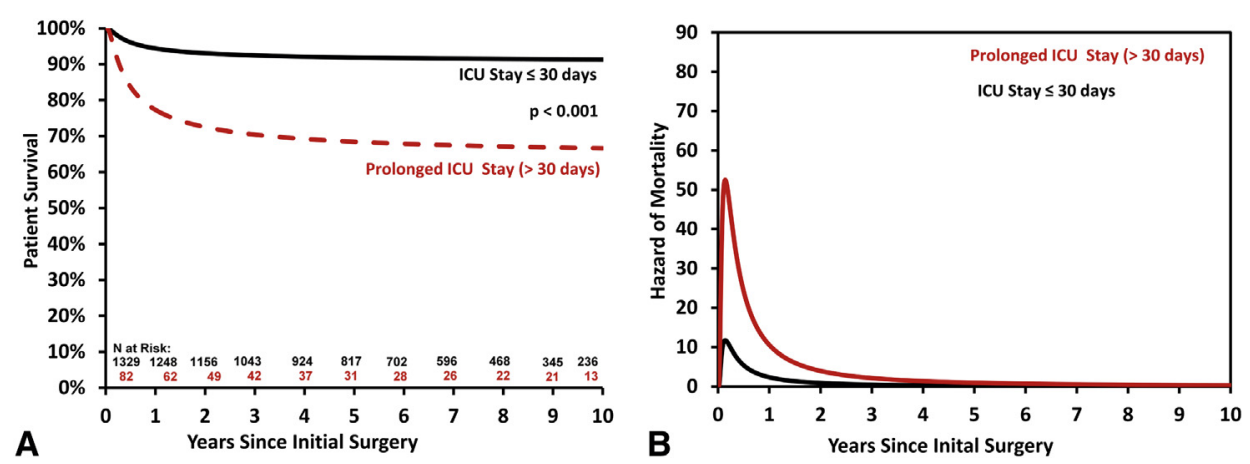

FIGURE 2. A and B, Parametric model for survival after index surgical procedure in neonates who survived initial hospitalization (hospital survivors ONLY) stratified by the requirement for prolonged postoperative ICU stay (yes vs no). ICU, Intensive care unit.

stay in the ICU to be more likely to die or require heart transplantation before Fontan $(18 \%$ vs $10 \%, P=.093)$.

\section{DISCUSSION}

In our experience, approximately $3 \%$ of all pediatric cardiac surgical patients required prolonged ICU stay $>30$ days, with the majority being neonates; approximately $7 \%$ of all neonatal cardiac surgical patients required prolonged ICU stay $>30$ days. Several previous studies have identified factors associated with prolonged ICU stay after pediatric cardiac surgery. Those factors included patient characteristics such as neonatal age, low weight, prematurity, genetic syndromes, and extracardiac malformations. ${ }^{10-17}$ Additional factors were related to underlying cardiac anomaly, such as the presence of single ventricle, and related to preoperative condition, need for preoperative mechanical ventilation, increased operative complexity, increased cardiopulmonary bypass time, and postoperative complications. $^{10-17}$ Our current findings are comparable

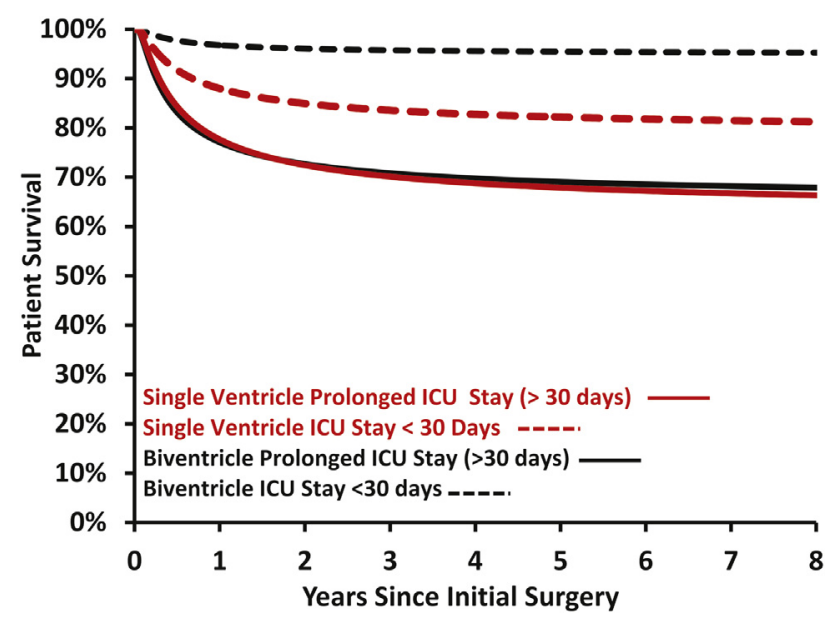

FIGURE 3. Parametric model for survival after index surgical procedure in neonates who survived initial hospitalization (hospital survivors ONLY) showing the interaction between underlying cardiac anomaly (single ventricle vs 2 ventricles) and the requirement for prolonged postoperative ICU stay (yes vs no). ICU, Intensive care unit. with those from previous studies, and the prevalence of prematurity, low weight, genetic syndromes, extracardiac malformations, and single-ventricle anomalies was significantly greater in neonates who required prolonged stay in the ICU. Similarly, neonates who required prolonged ICU stay were more likely to have needed unplanned cardiac reoperation or ECMO support. Those findings underscore the effect of residual lesions that have been linked to prolonged lengths of stay, increase in ventilation time, and mortality. ${ }^{17}$

Late outcomes beyond hospital discharge in pediatric cardiac surgery patients who required prolonged postoperative ICU stay have not been well explored. In a study that examined children with prolonged pediatric ICU stay via the Australia and New Zealand registry, children with cardiac anomalies comprised the second poorest long-term survival among the examined disease categories, with single-ventricle anomalies being an independent risk factor of death. ${ }^{18}$ In our surgical cohort, we demonstrated that there was an increase in mortality risk beyond hospital discharge with the majority of deaths occurring within the first year after the operation. Of importance, the vast majority of deaths were cardiac and many readmissions occurred after cardiac arrest with subsequent deterioration of status and patient demise. This finding highlights that the most important factor in determining late survival is the presence of residual cardiac lesions or myocardial dysfunction that will subject the patient to continual or recurrent deterioration of the cardiac status and consequently diminished survival.

We have examined the echocardiograms of patients who suffered from late mortality subsequent to hospital discharge. Although there were several patients who had residual lesions such as ventricular dysfunction, atrioventricular valve failure, pulmonary artery stenosis, etc, the presence of residual lesions was not found to be significantly associated with death in our series. Our analysis likely is limited in that regard because of the lack of universal definition of what constitutes a significant residual lesion, in addition to the small sample size from the statistical standpoint. Nonetheless, prolonged stay in the 


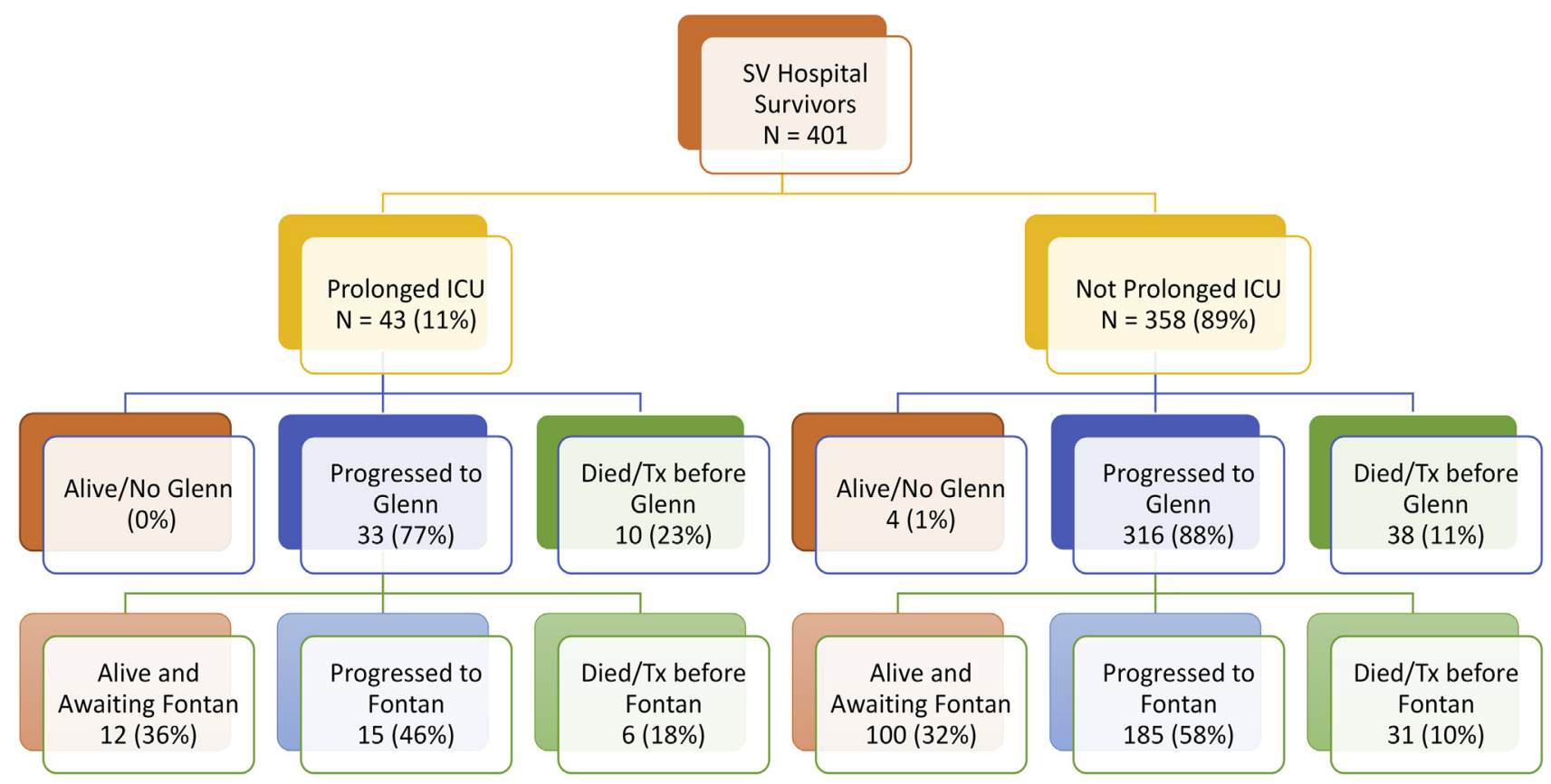

FIGURE 4. Flow chart demonstrating the progress to subsequent palliation stages in neonates with single ventricle after first-stage palliation surgery with comparison shown between neonates who required prolonged ICU stay after first-stage palliation versus not. Only hospital survivors are included. $S V$, Single ventricle; $I C U$, intensive care unit.

ICU should prompt physicians to identify and treat any important residual lesion that might have contributed to the complicated postoperative course early on to increase both hospital and late survival. This finding also suggests the need for a more vigilant postdischarge follow-up and the possible need for early assessment for candidacy for heart transplantation in those with myocardial dysfunction or residual lesions that are not amenable to surgical repair. In addition to cardiac deaths, a few patients died as the result of lingering multiorgan problems that were acquired during their complicated initial hospitalization, such as neurologic and lung injury.

We attempted to identify what postoperative complications or risk factors were associated with poor early or late outcomes. The 2 factors that were associated with hospital mortality were renal failure and need for ECMO. Our analysis might again be limited with this regard because of the overlap between the postoperative complications, with many patients suffering more than 1 complication, in addition to the small sample size from the statistical standpoint. Postoperative need for ECMO and prolonged duration of ECMO have been shown to be associated with prolonged stay in the ICU and early and late mortality. ${ }^{19-23}$ This continuous mortality risk may be caused by the prolonged effect of insults incurred by surgery and postoperative intensive care stay or may simply reflect the severity of those patients' conditions. Regardless of the cause, patients who required prolonged stay in the ICU should be considered as a high-risk group and would likely benefit from more rigorous work up to identify residual lesions and close follow-up and monitoring at home. Although many of those patients possessed established factors that would categorize them as high risk, prolonged stay in the ICU should be considered as an independent risk factor that should draw an additional caution.

An interesting and important finding in our study was that despite the negative effect of prolonged stay in the ICU on late outcomes was evident in all patients, that effect was more prominent in patients with 2 ventricles than in those with single ventricle. Despite the greater hospital mortality in single-ventricle patients, neonates with 2 ventricles who required prolonged postoperative ICU stay had more attrition beyond hospital discharge and in fact their late survival was equal to that for neonates with singleventricle anomalies. Taking into consideration that survival in neonates with 2 ventricles generally is superior to that with single ventricle, this interesting finding of comparable late outcomes suggests a more pronounced deviation from expected survival in children with 2 ventricles requiring prolonged postoperative ICU stay. This finding may reflect the fact that those patients with 2 ventricles might be sicker or have more residual lesions to require prolonged ICU stay because their expected requirement for ICU care should be shorter than that for single-ventricle patients. It is also plausible that single-ventricle patients may have experienced a more rigorous follow-up, given the need for subsequent surgical palliation, in which case would again 
suggest the need for more intensive monitoring of all those patients after hospital discharge.

In children with underlying single-ventricle anomalies, prolonged stay in the ICU was associated with high hospital mortality and increased interstage mortality before Glenn shunt. Nonetheless, the progression pattern after Glenn did not seem to be significantly affected, with $82 \%$ of patients receiving or qualifying for the subsequent Fontan operation.

In our study, we identified the effect of stay in the ICU as a continuous variable on late survival in all neonates who survived to hospital discharge after their initial neonatal cardiac surgery. We found that longer stay in the ICU was associated with worse survival in those patients. When we divided our patient population to 2 groups, we used the 30-day cutoff based on the work presented in previous studies in the pediatric and adult population that defined chronic critical illness as ICU stay more than 28-30 days. This cutoff has not been validated yet in the pediatric cardiac population. Our finding of worse outcomes in neonates with 2 ventricles suggest that what should be considered prolonged ICU stay for neonates with single ventricle might not necessarily be the same for those with 2 ventricles, who generally are expected to require shorter ICU stay. In studies on adult population who underwent cardiac surgery and experienced prolonged ICU stay, long-term survival and quality of life was poor, with $80 \%$ postdischarge mortality at 6 years and only $7 \%$ of the survivors regaining full function. ${ }^{3}$ On this basis, the authors have argued that the expensive and exhausting efforts to sustain those patients' lives may not be justifiable. The considerations for resource allocation cannot be overlooked in the pediatric population, because those patients require tremendous resources. ${ }^{9}$ Although caring for those patients is resource intensive, our data demonstrated greater late survival than that reported for adults.

Furthermore, given the multitude of factors that affect survival in those patients, we were unable to necessarily identify a pattern predictive of futile outcomes, short of renal failure requiring dialysis, to warrant early termination of care. Nonetheless, we have identified that prolonged stay in the ICU should be regarded as a marker for increased risk of late mortality and therefore approaches to identify and possibly treat modifiable factors could potentially improve prognosis. Finally, the association between prolonged stay in the ICU and quality of life in pediatric cardiac patients after discharge is not known and needs to be further determined to better understand the tangible prognosis of those challenging patients and help making clinical and administrative decisions.

\section{SUMMARY}

Inherent patient, anatomic, and postoperative risk factors are prevalent in neonates who require prolonged stay in the ICU after cardiac surgery. Prolonged postoperative stay in the ICU is associated with high hospital and significant postdischarge mortality, mainly during the first year. In neonates with underlying single-ventricle anomalies, prolonged stay in the ICU is associated with high hospital and interstage mortality and usual progression subsequent to Glenn shunt. On the other hand, prolonged ICU stay after surgery in patients with 2 ventricles anomalies is associated with high hospital mortality and considerable decrease in late survival, suggesting a more pronounced deviation from expected survival in children with 2-ventricle anomalies requiring prolonged postoperative ICU stay.

\section{Conflict of Interest Statement}

Authors have nothing to disclose with regard to commercial support.

\section{References}

1. Lagercrantz E, Lindblom D, Sartipy U. Survival and quality of life in cardiac surgery patients with prolonged intensive care. Ann Thorac Surg. 2010;89: 490-5.

2. Bapat V, Allen D, Young C, Roxburgh J, Ibrahim M. Survival and quality of life after cardiac surgery complicated by prolonged intensive care. J Card Surg. 2005;20:212-7.

3. Gaudino M, Girola F, Piscitelli M, Martinelli L, Anselmi A, Della Vella C, et al. Long-term survival and quality of life of patients with prolonged postoperative intensive care unit stay: unmasking an apparent success. J Thorac Cardiovasc Surg. 2007; 134:465-9.

4. Grothusen C, Attmann T, Friedrich C, Freitag-Wolf S, Haake N, Cremer J, et al. Predictors for long-term outcome and quality of life of patients after cardiac surgery with prolonged intensive care unit stay. Interv Med Appl Sci. 2013; 5:3-9.

5. Joskowiak D, Kappert U, Matschke K, Tugtekin S. Prolonged intensive care unit stay of patients after cardiac surgery: initial clinical results and follow-up. Thorac Cardiovasc Surg. 2013;61:701-7.

6. Gaies MG, Gurney JG, Yen AH, Napoli ML, Gajarski RJ, Ohye RG, et al. Vasoactive-inotropic score as a predictor of morbidity and mortality in infants after cardiopulmonary bypass. Pediatr Crit Care Med. 2010;11:234-8.

7. Ettema RG, Peelen LM, Schuurmans MJ, Nierich AP, Kalkman CJ, Moons KG. Prediction models for prolonged intensive care unit stay after cardiac surgery: systematic review and validation study. Circulation. 2010;122:682-9.

8. Messaoudi N, De Cocker J, Stockman BA, Bossaert LL, Rodrigus IE. Is EuroSCORE useful in the prediction of extended intensive care unit stay after cardiac surgery? Eur J Cardiothorac Surg. 2009;36:35-9.

9. Pasquali SK, He X, Jacobs ML, Shah SS, Peterson ED, Gaies MG, et al. Excess costs associated with complications and prolonged length of stay after congenital heart surgery. Ann Thorac Surg. 2014;98:1660-6.

10. Pagowska-Klimek I, Pychynska-Pokorska M, Krajewski W, Moll JJ. Predictors of long intensive care unit stay following cardiac surgery in children. Eur J Cardiothorac Surg. 2011;40:179-84.

11. Alsoufi B, McCracken C, Ehrlich A, Mahle WT, Kogon B, Border W, et al. Single ventricle palliation in low weight patients is associated with worse early and midterm outcomes. Ann Thorac Surg. 2015;99:668-76.

12. Kalfa D, Krishnamurthy G, Duchon J, Najjar M, Levasseur S, Chai P, et al. Outcomes of cardiac surgery in patients weighing $<2.5 \mathrm{~kg}$ : affect of patientdependent and -independent variables. J Thorac Cardiovasc Surg. 2014;148: 2499-506.

13. Alsoufi B, Gillespie S, Mahle W, Deshpande S, Kogon B, Maher K, et al. The impact of non-cardiac and genetic abnormalities on outcomes following neonatal congenital heart surgery. Semin Thorac Cardiovasc Surg. November 17, 2015 [Epub ahead of print].

14. Costello JM, Pasquali SK, Jacobs JP, He X, Hill KD, Cooper DS, et al. Gestational age at birth and outcomes after neonatal cardiac surgery: an analysis of the Society of Thoracic Surgeons Congenital Heart Surgery Database. Circulation. 2014;129:2511-7.

15. Brown KL, Ridout DA, Goldman AP, Hoskote A, Penny DJ. Risk factors for long intensive care unit stay after cardiopulmonary bypass in children. Crit Care Med. 2003;31:28-33. 
16. Alsoufi B, Manlhiot C, Mahle WT, Kogon B, Border WL, Cuadrado A, et al. Low-weight infants are at increased mortality risk after palliative or corrective cardiac surgery. J Thorac Cardiovasc Surg. 2014;148:2508-14.e1.

17. Nathan M, Karamichalis JM, Liu H, Emani S, Baird C, Pigula F, et al. Intraoperative adverse events can be compensated by technical performance in neonates and infants after cardiac surgery: a prospective study. J Thorac Cardiovasc Surg. 2011;142:1098-107, 1107 e 1-5

18. Namachivayam SP, Alexander J, Slater A, Millar J, Erickson S, Tibballs J, et al. Five-year survival of children with chronic critical illness in Australia and New Zealand. Crit Care Med. 2015;43:1978-85.

19. Gupta P, Robertson MJ, Beam B, Gossett JM, Schmitz ML, Carroll CL, et al. Relationship of ECMO duration with outcomes after pediatric cardiac surgery: a multi-institutional analysis. Minerva Anestesiol. 2015;81:619-27.

20. Alsoufi B, Al-Radi OO, Gruenwald C, Lean L, Williams WG, McCrindle BW, et al. Extra-corporeal life support following cardiac surgery in children: analysis of risk factors and survival in a single institution. Eur J Cardiothorac Surg. 2009;35:1004-11.
21. Lequier L, Joffe AR, Robertson CM, Dinu IA, Wongswadiwat Y, Anton NR, et al Two-year survival, mental, and motor outcomes after cardiac extracorporea life support at less than five years of age. J Thorac Cardiovasc Surg. 2008;136: 976-83.

22. Chan T, Thiagarajan RR, Frank D, Bratton SL. Survival after extracorporeal cardiopulmonary resuscitation in infants and children with heart disease. $J$ Thorac Cardiovasc Surg. 2008;136:984-92.

23. Alsoufi B, Al-Radi OO, Nazer RI, Gruenwald C, Foreman C, Williams WG, et al Survival outcomes after rescue extracorporeal cardiopulmonary resuscitation in pediatric patients with refractory cardiac arrest. J Thorac Cardiovasc Surg. 2007; 134:952-9.

Key Words: congenital heart disease, single ventricle, extracorporeal membrane oxygenation

\title{
EDITORIAL COMMENTARY
}

\section{Neonatal outcomes and length of stay: A firm grasp of the obvious?}

\author{
David M. Overman, MD
}

\footnotetext{
From the Division of Cardiac Surgery, The Children's Heart Clinic and Division of Cardiovascular Surgery, Children's Hospitals and Clinics of Minnesota, Minneapolis, Minn.

Disclosures: Author has nothing to disclose with regard to commercial support.

Received for publication May 27, 2016; accepted for publication May 31, 2016; available ahead of print July 1, 2016.

Address for reprints: David M. Overman, MD, Division of Cardiovascular Surgery, The Children's Heart Clinic,

2530 Chicago Ave South, Suite 500, Minneapolis, MN, 55404 (E-mail: doverman@chc-pa.org).

J Thorac Cardiovasc Surg 2016;152:727-8

$0022-5223 / \$ 36.00$

Copyright (c) 2016 by The American Association for Thoracic Surgery

http://dx.doi.org/10.1016/j.jtcvs.2016.05.061
}

Mori and colleagues' analysis of the association of intensive care unit (ICU) length of stay and outcomes in neonates after congenital heart surgery, ${ }^{1}$ on the surface at least, seems to state the obvious: if a newborn is in the ICU for an extended period, they are a member of a subgroup of patients who are at increased risk of mortality. It is tempting to set the authors' research aside with that observation, concluding there is little to be learned here. That would be unfortunate, however, as this manuscript offers more nuanced, and in some respects surprising insights into an association most of us would take for granted.

First, while an association between ICU length of stay and mortality is firmly established conceptually, the magnitude of this phenomenon is quite sobering. One in four of these patients, no matter the underlying anatomy, will die in the hospital and nearly half will die within

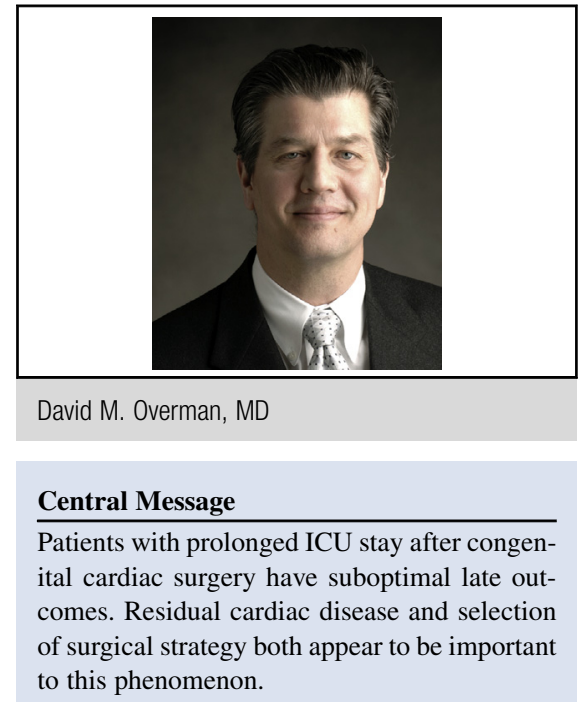

See Article page 720 .

1 year (using ICU admission as time zero). Of those who survive to hospital discharge, only two-thirds are alive at 1 year, compared with $92 \%$ for those who did not experience prolonged ICU stay. Thus, these data do not simply state the obvious; they emphatically highlight a subgroup of patients whose mortality risk (double that of the national average for a patient undergoing a 
TABLE E1. Univariate analysis of factors associated with survival subsequent to hospital discharge in neonates who required prolonged postoperative stay in the ICU

\begin{tabular}{lcc}
\hline \multirow{2}{*}{ Characteristic } & \multicolumn{1}{c}{ Univariate } \\
\cline { 2 - 3 } & Hazard ratio (95\% CI) & P value \\
\hline Male sex & $1.58(0.72-3.48)$ & .26 \\
Age at surgery & $0.97(0.91-1.04)$ & .37 \\
Premature $\leq 36 \mathrm{wk}$ & $1.40(0.58-3.33)$ & .45 \\
Genetic syndromes and & $1.90(0.86-4.19)$ & .11 \\
$\quad$ & & \\
$\quad$ Wextracardiac malformations & $0.79(0.27-2.28)$ & .66 \\
Two-ventricle anomaly & $0.97(0.45-2.09)$ & .94 \\
Cardiopulmonary bypass use & $1.32(0.45-3.82)$ & .61 \\
Postoperative ECMO use & $0.47(0.14-1.56)$ & .22 \\
Unplanned reoperation & $1.01(0.38-2.67)$ & .99 \\
STAT category 4 and 5 & $1.13(0.73-1.74)$ & .57 \\
Sepsis & $1.53(0.71-3.34)$ & .28 \\
Renal failure & $0.32(0.04-2.37)$ & .27 \\
Cerebrovascular accident & $1.28(0.30-5.40)$ & .74 \\
Necrotizing enterocolitis & $1.73(0.70-4.32)$ & .24 \\
Gastrointestinal bleeding & $1.74(0.24-12.84)$ & .59 \\
Chylothorax & $1.11(0.45-2.76)$ & .83 \\
Vocal cord paralysis & $1.48(0.35-6.27)$ & .59 \\
\hline$C I$, Confidence interval; $E C M O$, extracorporeal membrane oxygenation; STAT, Soci- \\
ety of Thoracic Surgeons-European Association for Cardio-Thoracic & Surgery \\
Congenital Heart Surgery mortality categories. &
\end{tabular}

